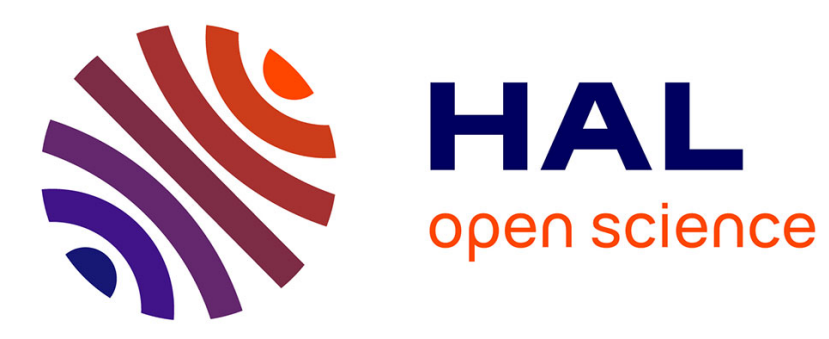

\title{
Active Mamyshev regenerator
}

Christophe Finot, Julien Fatome, Stéphane Pitois, Guy Millot, Erwan Pincemin

\section{To cite this version:}

Christophe Finot, Julien Fatome, Stéphane Pitois, Guy Millot, Erwan Pincemin. Active Mamyshev regenerator. Optical Review, 2011, 18 (3), pp.257-263. 10.1007/s10043-011-0052-9 . hal-00596244

\section{HAL Id: hal-00596244 \\ https://hal.science/hal-00596244}

Submitted on 29 May 2011

HAL is a multi-disciplinary open access archive for the deposit and dissemination of scientific research documents, whether they are published or not. The documents may come from teaching and research institutions in France or abroad, or from public or private research centers.
L'archive ouverte pluridisciplinaire HAL, est destinée au dépôt et à la diffusion de documents scientifiques de niveau recherche, publiés ou non, émanant des établissements d'enseignement et de recherche français ou étrangers, des laboratoires publics ou privés. 


\title{
Active Mamyshev Regenerator
}

\author{
Christophe FINOT $^{1}{ }^{*}$, Julien FATOME $^{1}$, Stéphane PITOIS $^{1}$, Guy MILLOT ${ }^{1}$ \\ and Erwan PINCEMIN ${ }^{2}$ \\ ${ }^{1}$ Laboratoire Interdisciplinaire CARNOT de Bourgogne,
} UMR 5209 CNRS/Université de Bourgogne, 9 av. Alain Savary, 21078 Dijon France

${ }^{2}$ France Telecom, Orange Labs, 2 Avenue Pierre Marzin, 22307 Lannion Cedex, France

In this contribution, we numerically and experimentally investigate the extension of the so-called Mamyshev regenerator with the implementation of an additional Raman gain. We evaluate the potential efficiency and advantages of this new active Mamyshev regenerator at $40 \mathrm{Gbit} / \mathrm{s}$ and we highlight a strong reduction of the working power as well as a large improvement of the available output power.

KEYWORDS : optical regeneration, nonlinear optics, optical fibers

*e-mail adress : christophe.finot@u-bourgogne.fr 


\section{Introduction}

With the development of long haul photonic networks working at high repetition rates (40 Gbit/s and beyond), performing an all-optical regeneration has become of a great interest to combat the cumulative impairments occurring during the signal propagation and to overcome the bandwidth limitations of the current electronic devices ${ }^{1)}$. Indeed, during its propagation, the signal undergoes various degradations such as amplified spontaneous emission noise accumulation, chromatic dispersion as well as intra-channel non-linear effects. Combination of all those effects translates into timing jitter, degradation of the extinction ratio or into detrimental amplitude jitter on the "one"-bit level of the signal ${ }^{2)}$. The latest two impairments can be partly overcome using $2 \mathrm{R}$ regeneration (Reamplification and Reshaping), in which a device performs optical limiting function and extinction ratio enhancement. This important building block of future high-speed optical grooming switches ${ }^{3)}$ can be achieved by using various optical methods taking advantage of the instantaneous Kerr non-linear response of optical fibers : non-linear optical loop mirrors (NOLMs) ${ }^{4,5)}$, four-wave mixing ${ }^{6)}$, or self-phase modulation (SPM) in normally ${ }^{7)}$ or anomalous ${ }^{8,9)}$ dispersive fibers (the two latest methods being known as "Mamyshev" (MR) and “spectrally filtered solitons" respectively). Thanks to its ability to simultaneously improve ' 0 ' and ' 1 ' bits of signal in a rather simple experimental scheme, MR technique has stimulated a large amount of works, both theoretical ${ }^{10-19)}$ and experimental ${ }^{7,12,16,19-22)}$.

Recent extensions have also been proposed in order to process several wavelengths ${ }^{23,24)}$ or polarizations ${ }^{25)}$ simultaneously, in order to gain some flexibility on the choice of the nonlinear fibers that are used ${ }^{26)}$ or in order to optimize the incoming pulse shape ${ }^{27)}$. Similarly to the nonlinear amplifying loop mirror ${ }^{28)}$ that is an evolution of a NOLM, another potential improvement that has been suggested to MRs is to combine physical amplification with nonlinear propagation ${ }^{29)}$.

In the present contribution, we give further details on this latest aspect by describing several advantages of using such a configuration. After describing our model, we will detail the improvements that can be expected on the transfer function and more specifically we will outline the reduced incoming power that is required as well as the improved output power. Qualitative results obtained based on a simplified model assuming constant gain will be confirmed by more realistic numerical simulations taking into account longitudinal pump depletion effects. We will finally illustrate and confirm our approach by experiments carried out at a repetition rate of 40 Gbits. 


\section{Principle of Operation and Configuration under Study}

A schematic of the active Mamyshev regenerator is shown in Fig. 1. The incoming pulsed signal is first amplified by an Erbium Doped Fiber Amplifier (EDFA) in order to reach the adequate peak power $P_{m}$ required for optimal peak power equalization of the 'one' symbols. The degraded signal then propagates in a Raman fiber amplifier (RFA) ${ }^{29)}$ based on a normally dispersive fiber which has a chromatic dispersion $\beta_{2}$, a nonlinear coefficient $\gamma$, and an optical distributed gain $g$. At the fiber output, a Gaussian optical bandpass filter (OBPF) with a FWHM spectral width $\delta$, is spectrally offset by an amount $\Delta \lambda$ with respect to the input signal carrier wavelength $\lambda_{0}$ and is used to carve into the broadened spectrum - thereby acting as a strong pulse reshaper.

The main point in the proposed configuration is the presence of a distributed gain in the nonlinear stage. Indeed, it has been demonstrated that any initial waveform propagating under conditions of normal dispersion, non-linearity and distributed gain will evolve asymptotically into a similariton, a pulse characterized by a parabolic intensity profile with a linear positive chirp ${ }^{30-}$ ${ }^{32)}$. Once this typical parabolic profile is obtained, it propagates self-similarly, i.e. the pulse is able to maintain its parabolic shape over propagation and to resist to the consequences of wavebreaking that usually induces strong unwanted distortions ${ }^{33)}$. The characteristic parameters of the similariton (peak power, temporal and spectral widths) then undergo an exponential increase with the propagation distance. A remarkable feature that constitutes a clear advantage for regeneration purpose $^{29)}$ is that this asymptotic profile does only depend on the initial pulse energy and not on the details of the initial pulse shape ${ }^{30,34)}$.

In the present paper, we discuss the impact of the use of the RFA. In order to illustrate our study, we consider the following parameters which are related to the experimental demonstration described in section 4 . The incoming pulses are chirp-free hyperbolic-secant pulses with an initial full-width at half maximum (FWHM) of $3.7 \mathrm{ps}$. The properties of the fiber are the following : $\beta_{2}=9.510-4 \mathrm{ps}^{2} / \mathrm{m}, \gamma=1010-3 / \mathrm{W} / \mathrm{m}$ and a length $\mathrm{L}=1 \mathrm{~km}$. As we have considered dispersion flattened fibers or fibers with a high $\beta_{3} / \beta_{2}$ ratio, we have neglected the influence of the third order dispersion $\beta_{3}$ for the sake of simplicity. The RFA exhibits an integrated gain $G$ of $10 \mathrm{~dB}$ and the output filter has a Gaussian shape with a FWHM spectral width of $100 \mathrm{GHz}$ and a frequency offset $\Delta \lambda$ of $200 \mathrm{GHz}$.

The performance of a $2 \mathrm{R}$ regenerator can primarily be assessed by its transfer function (TF) which relates the output to input pulse energies. Optimum power equalization occurs when the TF regime exhibits a plateau for the 'one' input level over a range of input pulse energies. As shown in ${ }^{11)}$, such a regime can be obtained when precise relationships between the input pulse, filter offset and normally dispersive fiber parameters are satisfied. In the context of this article, we do not intend to obtain such an optimum power equalization, therefore a non-monotonous TF 
(referred as A-type in ${ }^{11,35)}$ ) can be acceptable. Our main focus will be the input and output powers involved when working at the operating power.

\section{Comparison between Passive and Active Configurations}

\subsection{Pulse dynamics in a simplified model with constant distributed gain}

In order to get access to the $\mathrm{TF}$, we have first numerically simulated the propagation of a single optical pulse in the proposed regenerator ${ }^{11)}$. As a first approximation, the longitudinal evolution of the complex electric field envelope $\psi(\mathrm{z}, \mathrm{T})$ in the RFA can be modeled in terms of the wellknown nonlinear Schrödinger equation with gain term $g$ that is assumed to be longitudinally and spectrally constant ${ }^{31,32)}$ :

$$
i \frac{\partial \psi}{\partial z}=\frac{\beta_{2}}{2} \frac{\partial^{2} \psi}{\partial T^{2}}-\gamma|\psi|^{2} \psi+i \frac{g}{2} \psi
$$

Resulting TFs obtained for various values of the RFA integrated gain are plotted in Fig. 2. Two points can be noticed. First, the initial power required to obtain amplitude jitter improvement significantly decreases with increasing values of the gain (between $0 \mathrm{~dB}$ and $10 \mathrm{~dB}$ of gain, a reduction by a factor 4 is observed). Then, the power obtained after spectral filtering is enhanced (between $0 \mathrm{~dB}$ and $10 \mathrm{~dB}$ of gain, an increase by a factor 2.5 is recorded). From practical considerations, this implies that a reduction of the power of the EDFA is possible. Moreover, given the higher output power, no final additional amplification of the regenerated signal will be necessary, therefore limiting the potential amplified spontaneous emission in the system. Overall, the yield of the system as defined by the ratio between the outcoming and incoming power ${ }^{11)}$ is dramatically increased. These results clearly underline that the insertion of an additional distributed gain can potentially improve the Mamyshev regenerator performances.

In order to better understand the physical origin of the observed behavior, we have plotted in Fig. 3(a) the temporal intensity profiles after the nonlinear propagation in the passive or active HNLF and before spectral filtering (initial pulses having the initial power corresponding to the working points of the regenerator). The output pulse shape exhibit marked differences : the pulses obtained after propagation in the RFA clearly exhibits the parabolic signature ${ }^{31,32)}$. On the contrary, in the passive fiber, the temporal profile is flattened as can be obtained after the wavebreaking stage ${ }^{33)}$ and which has been found to be crucial for MR behavior ${ }^{11)}$.

From Fig. 3(a), one can also notice that pulses obtained in active architecture experience lower temporal broadening than the pulses in the passive configuration. This feature is beneficial in order to avoid the deleterious effect of sequence patterning that degrades the performance of MRs due to pulse to pulse interactions in the regenerator ${ }^{13,21,36)}$. This reduced output duration can be understood in view of the longitudinal temporal and spectral evolutions of the pulses (Fig. 3(b) and 3(c) respectively). In the case of passive configuration, most of the spectral 
broadening occurs at the beginning of the propagation, inducing through dispersion a temporal broadening of the pulse and therefore reducing their peak power ${ }^{33)}$. On the contrary, in active amplification, the peak power of the pulse increases with propagation length so that self-phase modulation becomes significant only in the second part of the fiber. Consequently, dispersion acts on the expanded spectrum over a reduced length, which explains the observed limited temporal broadening. We may also anticipate some beneficial consequences in terms of timing jitter of the regenerated pulse ${ }^{11,13)}$ : as can be observed in Fig. 3(a), the delay for both configuration is not identical.

\subsection{Realistic simulations}

The conclusions discussed in the previous subsection are based on the assumption of a constant gain, neglecting therefore potential depletion effects of the pump, which is fully justified in the case of a single pulse. When considering a train at a repetition rate of several tens of $\mathrm{GHz}$, the assumption is not fulfilled anymore and the gain exhibits a longitudinal evolution $g(z)$. In order to take this effect into account, we have performed realistic simulations relying on the commercial software VPI Transmission Maker.

The numerical simulations are based on the experimental data described in the last section. A 40-Gbit/s Return-to-Zero (RZ) Pseudo Random Bit Sequence (PRBS) signal made of 3.7-ps chirp-free hyperbolic-secant pulses is injected into a 1-km long highly nonlinear fiber whose properties have been described in section 2. After nonlinear propagation within the fiber and spectral broadening, a 100-GHz OBPF is used to carve in the resulting spectrum with a 400$\mathrm{GHz}$ offset filtering.

By varying the average power of the initial signal, we have calculated the transfer function of the device. From Fig. 4(a), we can make out that the various qualitative conclusions previously drawn in a single pulse configuration are valid. In particular, we clearly observe that increasing the pump power allows a much lower working power as well as an increased output power. From these simulations, we may also notice a small degradation of the extinction ratio of the device, but this degradation is artificial and is caused by depletion effects: the high average power pulse train undergoes lower amplification than a low average power one. Nevertheless, in practical situation (assuming a counter-propagative pumping), both low and high power pulses will undergo the same averaged gain.

In order to highlight the regeneration efficiency of our device on the intensity profile, we have performed numerical simulations on an artificially degraded 40-Gbit/s signal. At the input of our regenerator, the extinction ratio and amplitude jitter of the initial signal are voluntary degraded by means of two optical intensity modulators driven by random white Gaussian electrical noises. The resulting poor quality eye diagram of the 40-Gbit/s input signal is depicted 
in Fig. 4(b). After propagation in our regenerator with an input average power of $50 \mathrm{~mW}$ and a pump power of $1750 \mathrm{~mW}$, the quality of the eye diagram is clearly improved with a great increase of the eye-opening (Fig. 4(c)). The energy initially contained into the zero bit-slots is largely annihilated thanks to the offset filtering, while the amplitude jitter is greatly reduced by means of the inflexion point of the TF. However, we can notice the appearance of a small timing jitter, intrinsic to the Mamyshev-based regenerator ${ }^{13)}$.

\section{Proof of Principle Experiment}

\subsection{Experimental setup}

Our experimental set-up is sketched on Fig. 5. A modelocked fiber-based laser delivers a 10-GHz train of transform-limited picosecond pulses. The pulses have a FWHM duration of 3.7 ps and a central wavelength of $1550.1 \mathrm{~nm}$. The pulse train is then temporally multiplexed to obtain a $40 \mathrm{GHz}$ signal thanks to a $\mathrm{x} 4$ optical bit rate multiplier (BRM). This $40-\mathrm{GHz}$ signal is finally encoded through a 40-Gbit/s PRBS 4x10 Gbit/s multiplexed signal driving an optical modulator.

In order to simulate deleterious ghost pulses, we have voluntarily used a non-optimum bias in our modulator so as to degrade the extinction ratio of the initial pulse train. A second optical intensity modulator driven by a random white Gaussian electrical noise delivered by an arbitrary waveform generator simulates additional amplitude jitter on the one level of the pulses. An EDFA is then used in order to boost the average power to the required level. In order to maintain the level of potential nonlinear degradation in the EDFA identical for all the tested powers, the initial power is adjusted through the use of an optical variable attenuator (OVA). The regenerator is made of highly nonlinear fiber whose properties have been described in section 2 . Note that thanks to the reduced temporal duration of the initial pulses, deleterious consequences of Brillouin backscattering have been avoided and that no additional compression stage is required $^{21)}$.

The pumping of the lumped RFA is made by a continuous Raman fiber laser with a central wavelength of $1455 \mathrm{~nm}$, therefore ensuring a maximum gain in the conventional band of optical telecommunications. The maximum output power of the pump laser is $2 \mathrm{~W}$ and the delivered wave is partially coherent (the spectral width of the pump is $100 \mathrm{GHz}$ ) so that no Brillouin scattering has been observed. As our pump is unpolarized, our proposed architecture has been shown to be rather polarization insensitive. Our setup is based on a counter-propagative pumping scheme is order to limit the temporal broadening of the pulse in the amplifier ${ }^{37)}$. This also limits the relative noise intensity transfer from the pump to the amplified signal ${ }^{38)}$ and prevents the emergence of extreme events as well as effects of cross-phase modulation between the pump and the signal ${ }^{39}$ ). This requirement of counter-propagative configuration makes unfortunately the device unpractical for bidirectionnal operation ${ }^{24,25,40)}$. 
After the fiber, a wavelength demultiplexer is used in order to carve in the expanded spectrum. The spectral width $\delta f$ of the channel of the component is $100 \mathrm{GHz}$ and the different channels rely on an International Telecommunication Union (ITU) grid spacing of $200 \mathrm{GHz}$. Consequently, the spectral filtering that is involved corresponds to a wavelength conversion from standardized ITU channel $34(1550.116 \mathrm{~nm})$ to channel $36(1548.515 \mathrm{~nm})$.

\subsection{Experimental transfer functions}

By changing the initial average power of the incoming pulse train, we have measured the TFs for various pump powers. Results presented in Fig. 6(a) are in full agreement with the qualitative conclusions drawn in the previous section and in particular in good agreement with the numerical results of Fig. 4(a): for increasing pump powers, the required input power is lowered whereas the output power is increased. Normalizing the TF with respect to the working power parameters, we can also make out that the plateau has a wider extension (Fig. 6(b)), therefore enhancing the ' 1 ' level equalization properties of the device. However, our experimental measurements also stress a slight apparent degradation of the regenerated extinction ratio (Fig. 6(b2)). This point has however to be nuanced as the higher level of low power pulse train is an artifact due to the nondepletion of the pump. More rigorous analysis would therefore require the use of controllable ghost pulses such as used in ${ }^{29)}$.

More quantitative measurements of the input and output average powers of the pulse trains are summarized in Fig. 7(a). We experimentally observe a close to linear decrease and increase of the incoming and outcoming powers respectively (Figs. 7(a1) and 7(a2) respectively). All in all, the yield (Fig. 7(a3)) is significantly improved from a poor $1.7 \%$ in passive configuration up to $22 \%$ when a pump power of $1750 \mathrm{~mW}$ is applied. By varying the signal central wavelength, we have carried out additional measurements for $\Delta \lambda$ values of the frequency offset ranging between 150 and $500 \mathrm{GHz}$. Results are plotted on Fig. 7(b) and confirm that higher the frequency detuning is, higher is the required incoming average power needed to obtain power equalization ${ }^{11)}$, following a close to linear law when plotted on a semi-logarithmic scale. Moreover, the benefits of the active configuration demonstrated for an offset of $200 \mathrm{GHz}$ are preserved for other frequency offset values.

\subsection{Temporal and spectral characterizations}

We have complemented our TF measurements by spectral and temporal recording of the nonmodulated 10-GHz pulse train. Optical spectra of the incoming pulse are plotted on Fig. 8(a) and exhibit very good agreement with a hyperbolic secant fit. Subplots (b) outline the significant spectral broadening undergone by the pulse train. The spectral expanded shapes are very similar 
but we can notice a higher spectral density in the case of the active configuration. Note also that in passive configuration, spectral wings characteristics of wave-breaking are visible whereas such feature is less apparent in the self-similar parabolic RFA ${ }^{33)}$. At the output, the carved pulse is in clear agreement with the Gaussian shape of the wavelength demultiplexer.

To gain further insight on the temporal nature of the pulses, we have recorded the optical intensity autocorrelations of the initial and output pulses trains (Fig. 9). The results confirm the transform-limited nature of the initial pulses (with a time-bandwidth product of 0.33 ), in excellent agreement with a fit by a hyperbolic secant shape. After spectral slicing of the broadened spectrum, we can first notice a change of the shape of the autocorrelation, now in agreement with a Gaussian fit. Therefore, the regenerator acts as an efficient reshaper ${ }^{41)}$. The temporal duration is estimated to $5 \mathrm{ps}$, which is slightly longer than what can be expected from the Fourier limit (4.4 ps). This outlines that pulse emerging from MR exhibit a low residual chirp ${ }^{11)}$ that could have been efficiently removed by an additional short segment of fiber with anomalous dispersion. Let us note that no significant difference between the active and passive configurations has been observed regarding the temporal and spectral properties of the pulses obtained after regeneration, which confirms the trends also observed in Fig. 3(a) (dotted lines).

Finally, we have recorded the eye diagrams obtained for the degraded 40-Gbit/s RZ PRBS signal. Figure 10(a) clearly shows the poor extinction ratio of the initial signal as well high fluctuations of the ' 1 ' level. After regenerator, significant enhancement of the extinction ratio is achieved with much less energy contained in the ' 0 ' bit slots of information. Regarding the amplitude jitter, improvement is also remarkable. Initial root mean square amplitude jitter has indeed been estimated to $13 \%$ of the initial peak power. After passive regeneration, this value has fallen down to $7 \%$. If the active configuration is issued, this value decreases down to $6 \%$, which is consistent with the broadened plateau of the active MR.

\section{Conclusions}

We have described the various benefits of an active Mamyshev regenerator combining Raman amplification and nonlinear propagation in a normally dispersive fiber. The power required for a proper use of the device is significantly decreased whereas the output power is enhanced. Reduced temporal broadening in the fiber segment is attractive in order to reduce pulse to pulse interaction. The device has been experimentally demonstrated to provide wavelength conversion as well as temporal and shape modification.

Further optimization of the choice of the fiber properties may enable to improve the configuration in order to obtain a better power level equalization . Moreover, using a longer fiber may enable a higher Raman gain and therefore it may become possible to get rid of the initial EDFA, simplifying therefore the experimental setup. This would provide also very promising 
perspectives for optical regenerators out of the range of the conventional band of telecommunication when no efficient EDFA are available ${ }^{42)}$. Let us finally note that the presence of a physical gain is not a mandatory condition to observe the parabolic dynamics and that similar behavior can be also experienced in a passive normally dispersive fiber where a longitudinal decrease of the dispersion value may mimic the effect of distributed Raman gain ${ }^{43,44)}$.

\section{Acknowledgement}

This work is supported by the Agence Nationale de la Recherche (FUTUR ANR-06-TCOM-016, PERSYST II ANR-07-TCOM-014) and by the Conseil Régional de Bourgogne. Experiments have benefited from the PICASSO research platform. 


\section{Reference list}

1) O. Leclerc, B. Lavigne, E. Balmefrezol, P. Brindel, L. Pierre, D. Rouvillain, and F. Seguineau: J. Lightw. Technol. 21 (2003) 2779-2790.

2) G. P. Agrawal, Fiber-Optic Communication Systems (Wiley-Interscience, 2002).

3) P. Vorreau, S. Sygletos, F. Parmigiani, D. Hillerkuss, R. Bonk, P. Petropoulos, D. J. Richardson, G. Zarris, D. Simeonidou, D. Klonidis, I. Tomkos, R. Weerasuriya, S. Ibrahim, A. D. Ellis, D. Cotter, R. Morais, P. Monteiro, S. Ben Ezra, S. Tsadka, W. Freude, and J. Leuthold: Opt. Express 17 (2009) 15173-15185.

4) N. J. Doran and D. Wood: Opt. Lett. 13 (1988) 56-58.

5) S. Boscolo, S. K. Turitsyn, and K. J. Blow: Opt. Fiber. Technol. 14 (2008) 299-316.

6) E. Ciaramella, F. Curti, and S. Trillo: IEEE Photon. Technol. Lett. 13 (2001) 142-144.

7) P. V. Mamyshev, "All-optical data regeneration based on self-phase modulation effect," in European Conference on Optical Communication, ECOC'98, 1998), 475-476.

8) M. Asobe, A. Hirano, Y. Miyamoto, K. Sato, K. Hagimoto, and Y. Yamabayashi: Electron. Lett. 34 (1988) 1135-1136.

9) M. Gay, M. Costa e Silva, T. N. Nguyen, L. Bramerie, T. Chartier, M. Joindot, J. C. Simon, J. Fatome, C. Finot, and J. L. Oudar: IEEE Photon. Technol. Lett. 22 (2010) 158160.

10) T. N. Nguyen, M. Gay, L. Bramerie, T. Chartier, and J. C. Simon: Opt. Express 14 (2006) 1737-1747.

11) L. Provost, C. Finot, K. Mukasa, P. Petropoulos, and D. J. Richardson: Opt. Express 15 (2007) 5100-5113.

12) M. Rochette, L. B. Fu, V. G. Ta'eed, D. J. Moss, and B. J. Eggleton: IEEE J. Sel. Top. Quantum Electron. 12 (2006) 736-744.

13) C. Finot, T. N. Nguyen, J. Fatome, T. Chartier, L. Bramerie, M. Gay, S. Pitois, and J. C. Simon: Opt. Commun. 281 (2008) 2252-2264.

14) T. I. Lakoba: IEEE J. Sel. Top. Quantum Electron. 14 (2008) 599-609.

15) S. Pitois, C. Finot, and L. Provost: Opt. Lett. 32 (2007) 3263-3265.

16) T.-H. Her, G. Raybon, and C. Headley: IEEE Photon. Technol. Lett. 16 (2004) 200-202.

17) P. P. Baveja, D. N. Maywar, and G. P. Agrawal: J. Lightw. Technol. 27 (2009) 38313836.

18) A. G. Striegler and B. Schmauss: J. Lightw. Technol. 24 (2006) 2835-2843.

19) B. E. Olsson and D. J. Blumenthal: J. Lightw. Technol. 20 (2002) 1113-1117.

20) J. Leuthold, G. Raybon, Y. Su, R. J. Essiambre, S. Cabot, J. Jacques, and M. Kauer: Electron. Lett. 38 (2002) 890-891. 
21) T. N. Nguyen, T. Chartier, L. Bramerie, M. Gay, Q. T. Le, S. Lobo, M. Joindot, J. C. Simon, J. Fatome, and C. Finot: Opt. Express 17 (2009) 17747-17757.

22) H. Murai, Y. Kanda, M. Kagawa, and S. Arahira: J. Lightw. Technol. 28 (2009) 910-921.

23) M. Vasilyev and T. I. Lakoba: Opt. Lett. 30 1458-1460.

24) L. Provost, F. Parmigiani, C. Finot, K. Mukasa, P. Petropoulos, and D. J. Richardson: Opt. Express 16 (2008) 2264-2275.

25) A. L. Yi, L. S. Yan, B. Luo, W. Pan, J. Ye, and J. Leuthold: Opt. Express 18 (2010) 71507156.

26) L. Provost, C. Finot, P. Petropoulos, and D. J. Richardson: J. Lightw. Technol. 28 (2009) 1373-1379.

27) H. Goto, T. Konishi, and K. Itoh: J. Opt .A. 10 (2008) 095306.

28) M. E. Fermann, F. Haberi, M. Hofer, and H. Hochreiter: Opt. Lett. 15 (1990) 752-754.

29) C. Finot, S. Pitois, and G. Millot: Opt. Lett. 30 (2005) 1776-1778.

30) M. E. Fermann, V. I. Kruglov, B. C. Thomsen, J. M. Dudley, and J. D. Harvey: Phys. Rev. Lett. 84 (2000) 6010-6013.

31) C. Finot, J. M. Dudley, B. Kibler, D. J. Richardson, and G. Millot: IEEE J. Quantum Electron. 45 (2009) 1482-1489.

32) C. Finot, G. Millot, C. Billet, and J. M. Dudley: Opt. Express 11 (2003) 1547-1552.

33) C. Finot, B. Kibler, L. Provost, and S. Wabnitz: J. Opt. Soc. Am. B 25 (2008) 1938-1948.

34) C. Finot, G. Millot, and J. M. Dudley: Opt. Lett. 29 (2004) 2533-2535.

35) J. Fatome and C. Finot: J. Lightw. Technol. 28 (2010) 2552-2556.

36) C. Finot and G. Millot: Opt. Express 13 (2005) 5825-5830.

37) C. Finot: Opt. Commun. 249 (2005) 553-561.

38) C. Fludger, V. Handerek, and R. J. Mears: J. Lightw. Technol. 19 (2001) 1140-1148.

39) K. Hammani, C. Finot, J. M. Dudley, and G. Millot: Opt. Express 16 (2008) 1646716474.

40) M. Matsumoto, Y. Shimada, and H. Sakaguchi: IEEE J. Quantum Electron. 45 (2009) 5157.

41) C. Finot and G. Millot: Opt. Express 12 (2004) 5104-5109.

42) M. Matsuura and N. Kishi: Opt. Lett. 34 (2009) 2420-2422.

43) T. Hirooka and M. Nakazawa: Opt. Lett. 29 (2004) 498-500.

44) C. Finot, B. Barviau, G. Millot, A. Guryanov, A. Sysoliatin, and S. Wabnitz: Opt. Express 15 (2007) 15824-15835. 


\section{Figure captions}

Figure 1 : Active Mamyshev regenerator set-up

Figure 2 : Transfer function obtained for various values of the integrated gain $G$ of the Raman fiber amplifier.

Figure 3: (a) Temporal intensity of the pulse after propagation in the normally dispersive segment for active (solid black line) or passive (solid grey line) configurations. The initial pulse is plotted with circles and is compared with the output pulse obtained in both configurations (dotted grey and black lines). (b) Longitudinal evolution of the temporal profile in the passive and active configuration (subplot 1 and 2 respectively). (c) Longitudinal spectral evolution of the spectral profile in passive and active configurations (subplot $\mathbf{1}$ and $\mathbf{2}$ respectively)

Figure 4 : (a) 40-Gbit/s numerical results of the transfer function for various pump levels. The pump depletion is taken into account (b) Input eye diagram of the degraded 3.7-ps 40-Gbit/s PRBS signal (c) Corresponding regenerated signal for an input average power of $50 \mathrm{~mW}$ and a 1750-mW pump power.

Figure 5 : Experimental set-up. OM - Optical Modulator. PM - Powermeter. BRM - Bit Rate Multiplier. PRBS - Pseudo Random Bit Sequence. ARB. - Arbitrary. OVA - Optical Variable Attenuator. WDM - Wavelength Division Multiplexing. ITU - International Telecommunication Union.

Figure 6 : Experimental measurements of the transfer function for various pump levels. (a) non normalized TFs and (b) normalized TFs.

Figure 7 : (a) Working power, outcoming average power and yield for different values of the pump power (subplot $\mathbf{a 1}, \mathbf{a} 2$ and $\mathbf{a} 3$ respectively). (b) Required working power for passive and active configurations (black diamonds and full grey circles respectively). The pump power is $1750 \mathrm{~mW}$. The solid lines represent a linear fit of the values (when plotted on a semilogarithmic scale).

Figure 8 : Spectral properties obtained in the passive (subplots 1) and active (1750 mW pump power, subplots 2) configurations. Incoming signals (subplots (a)) are compared with an hyperbolic secant fit (solid black line). Spectra recorded before spectral carving are plotted in 
subplots (b). Spectrum obtained after the wavelength demultiplexer (subplots (c)) are compared with a Gaussian fit (solid black line).

Figure 9 : Experimental autocorrelation traces of the input and output signal (grey circles and black crosses respectively) in passive (a) and active (b) configuration. The experimental measurements are compared with hyperbolic secant (solid grey line) and Gaussian (solid black line) respectively.

Figure 10 : Eye diagram of the 40-Gbit/s RZ signal recorded before the regenerator (a) and after the regenerator based on a passive (b) or active for a pump power of $1750 \mathrm{~mW}$ (c) architecture. 


\section{Figure 1}

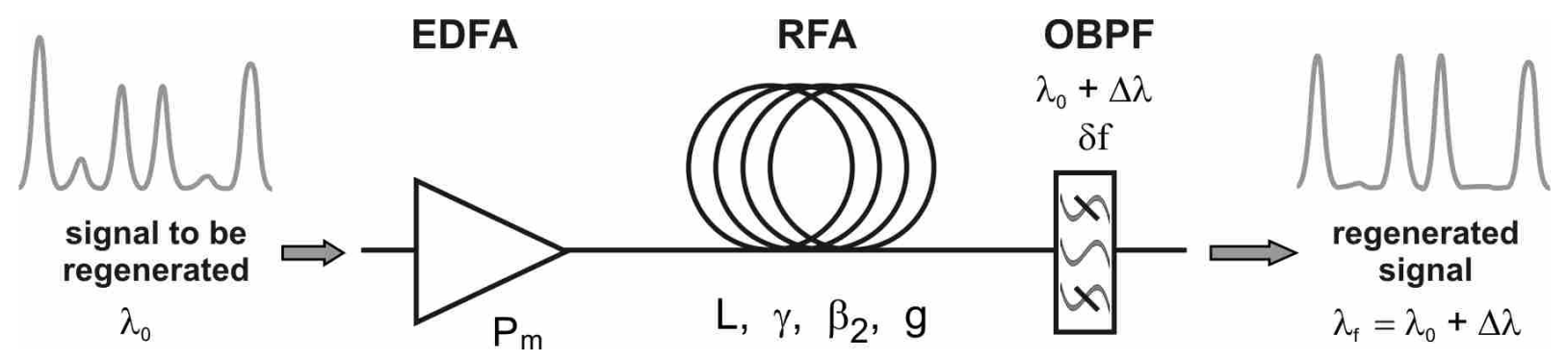


Figure 2

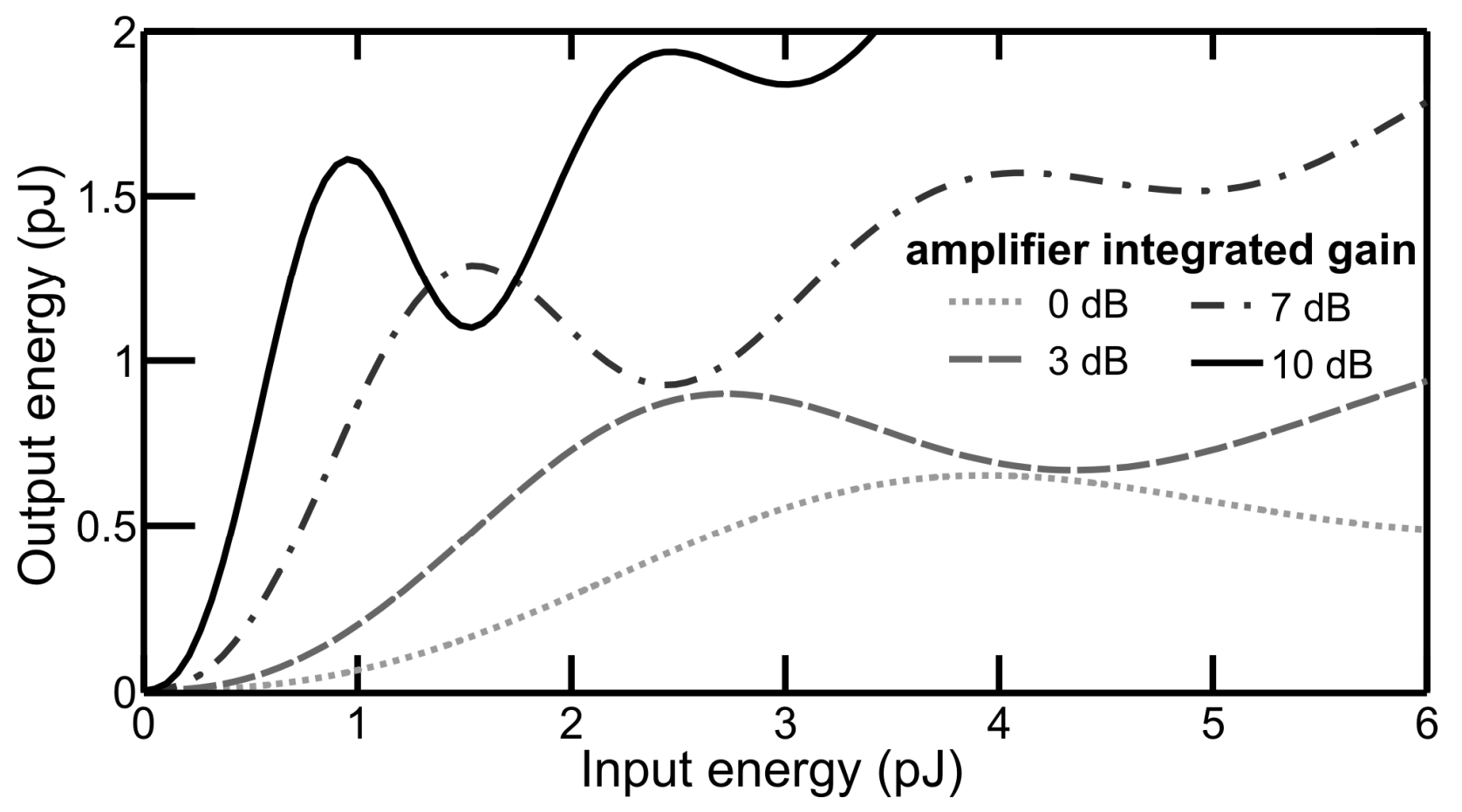


Figure 3
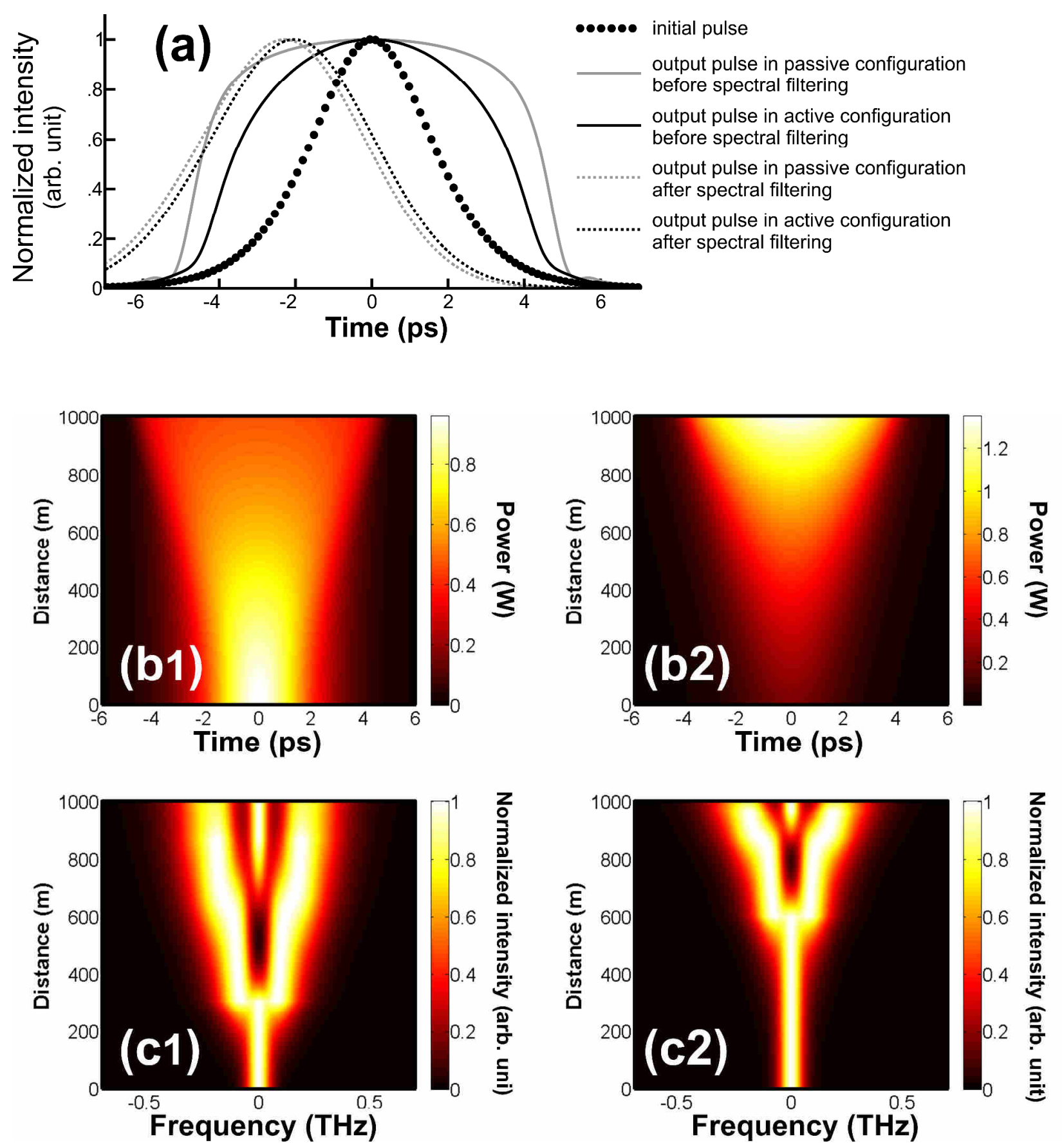
Figure 4
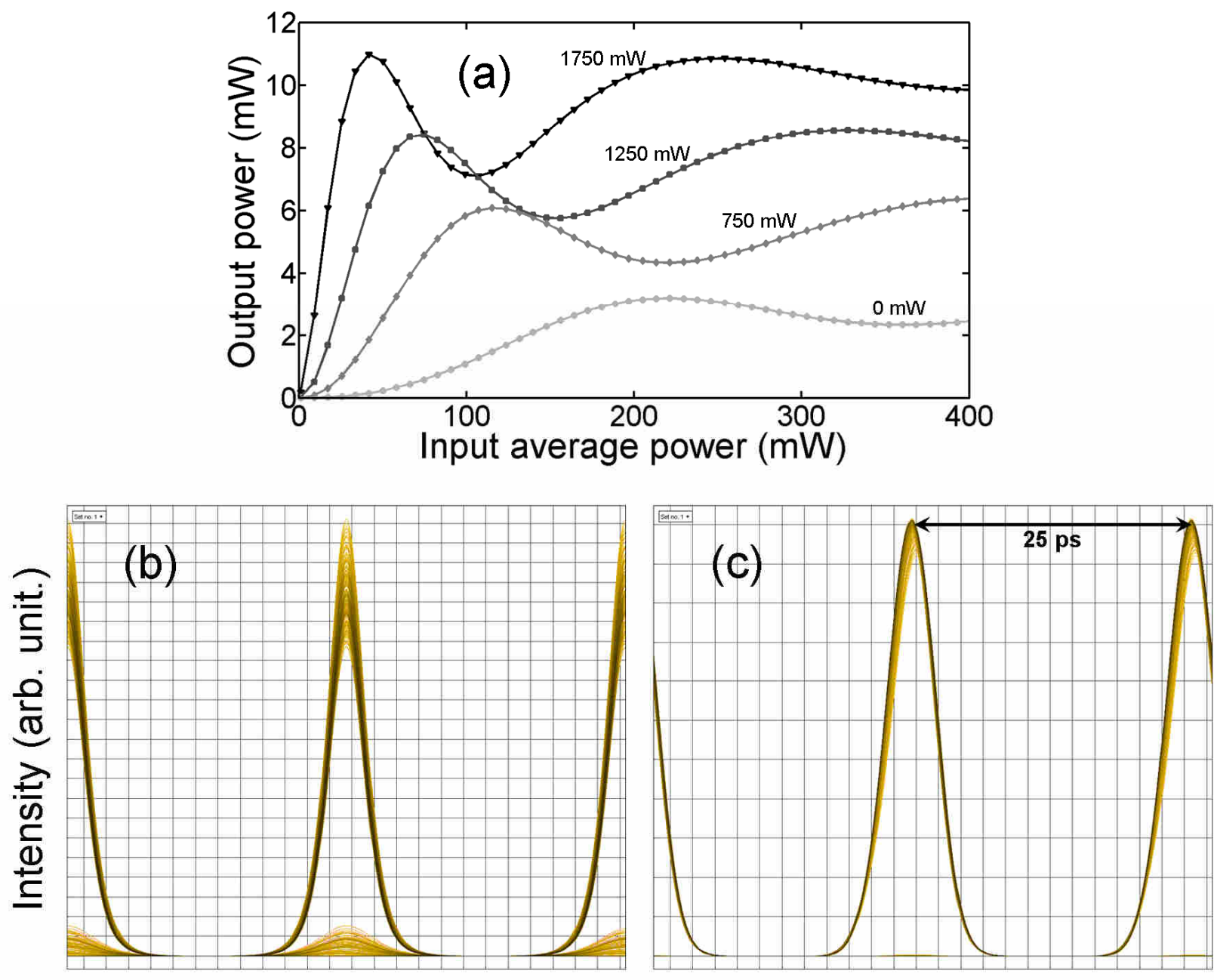
Figure 5

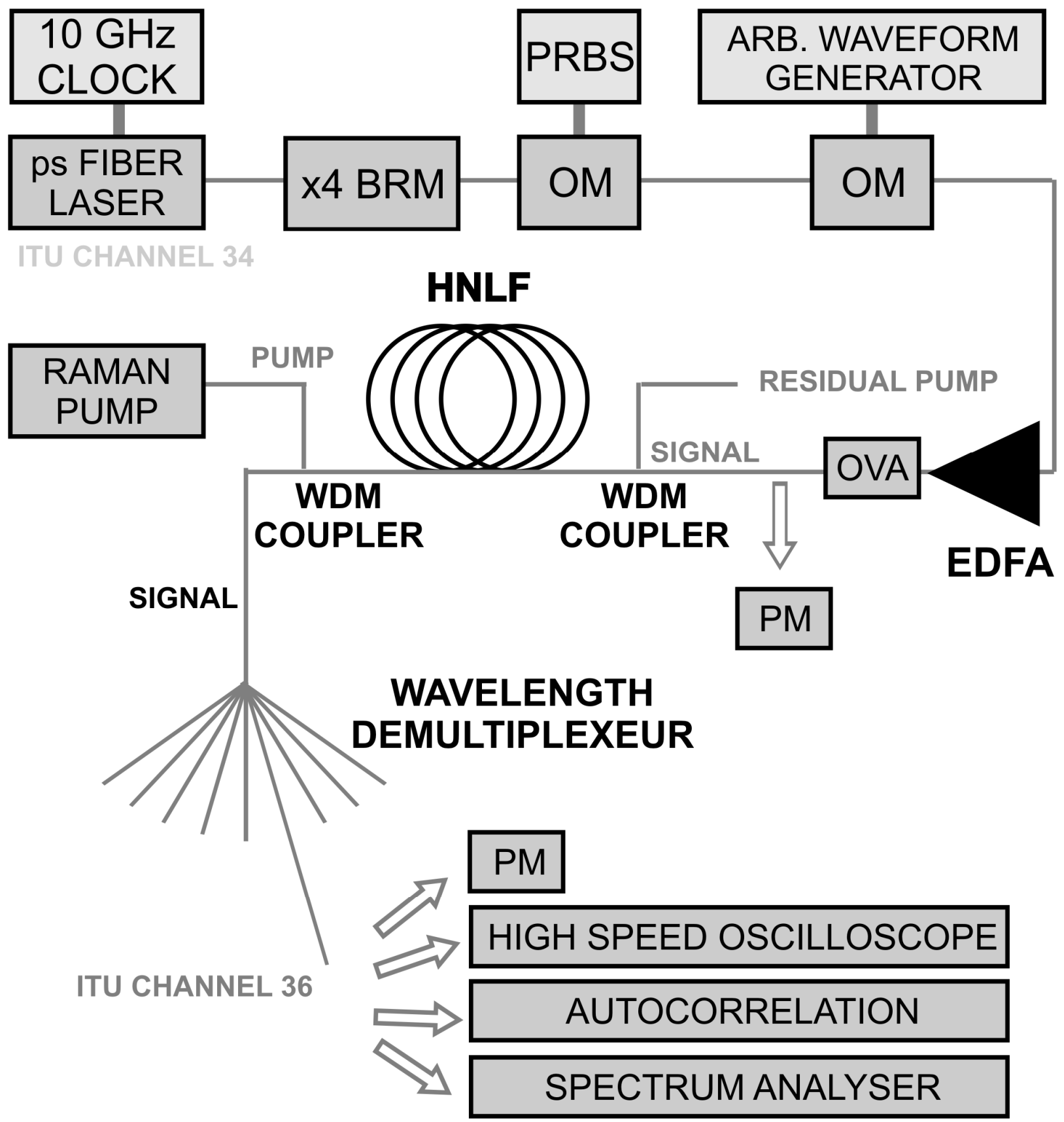


Figure 6

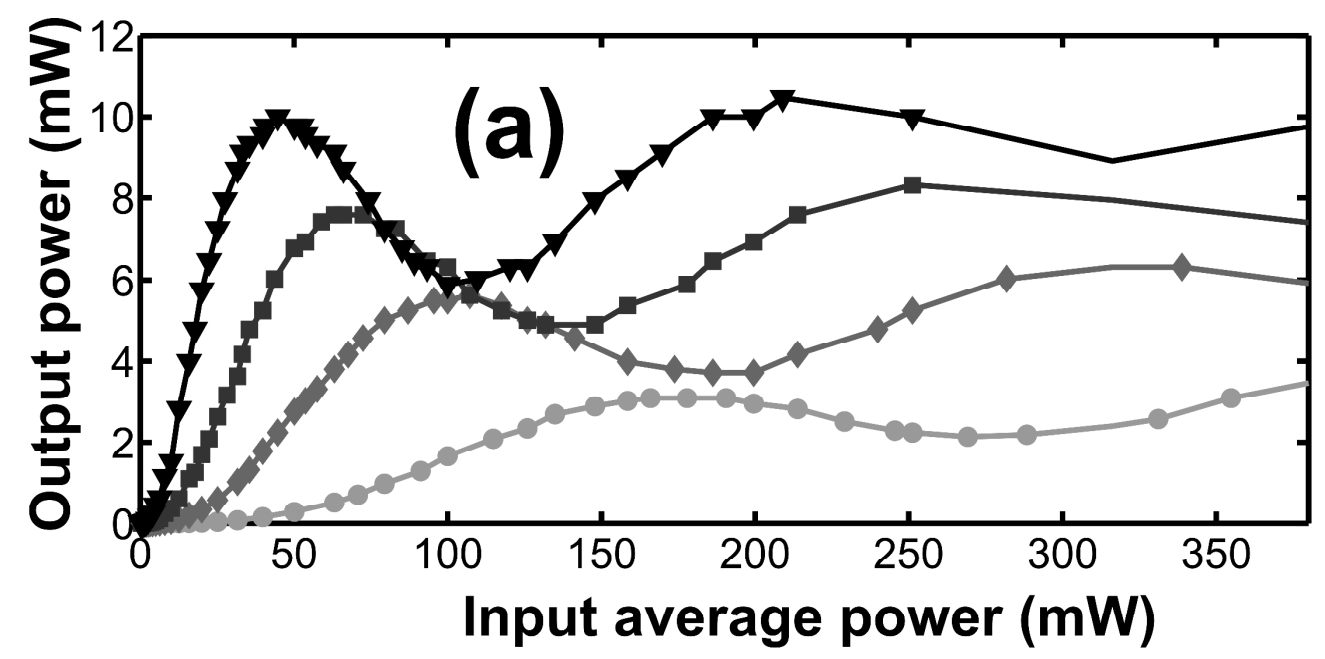

RFA

pump power :

、 $1750 \mathrm{~mW}$

- $1250 \mathrm{~mW}$

- $750 \mathrm{~mW}$

- $0 \mathrm{~mW}$

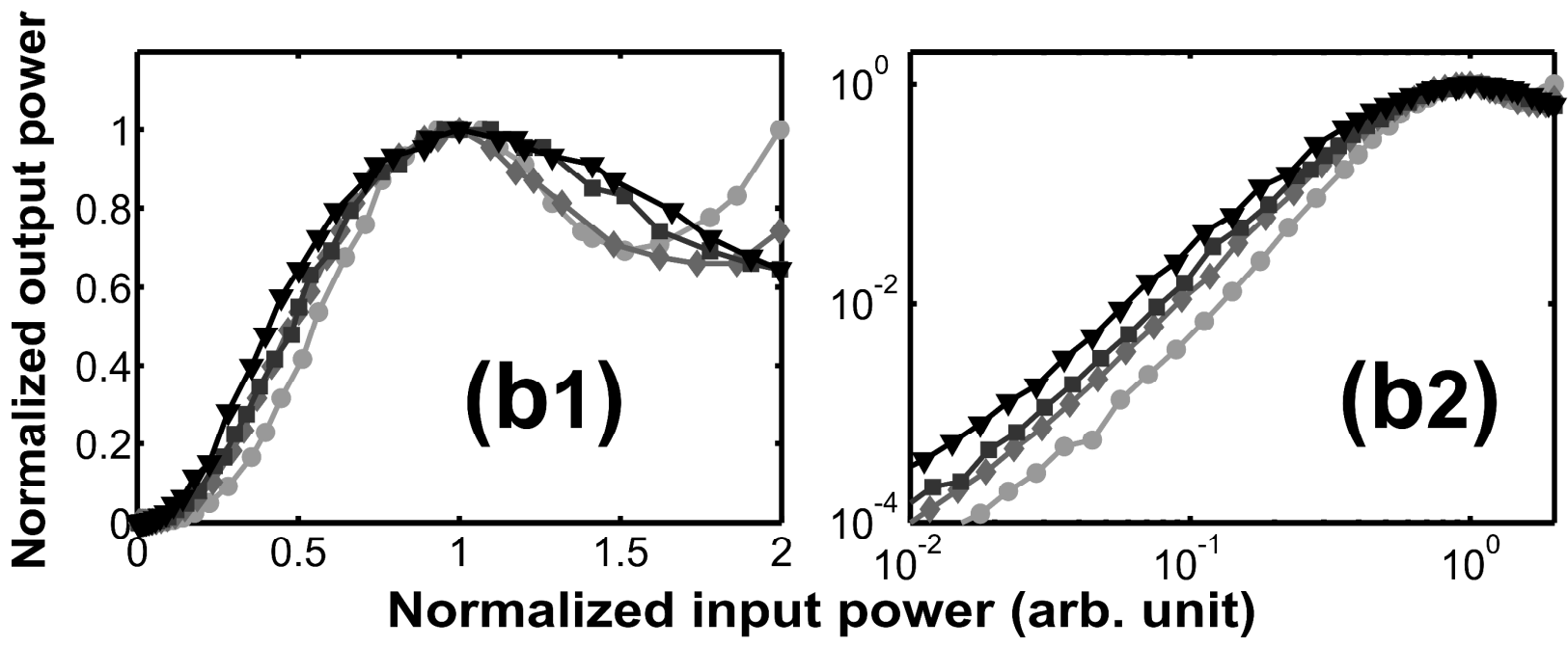


Figure 7
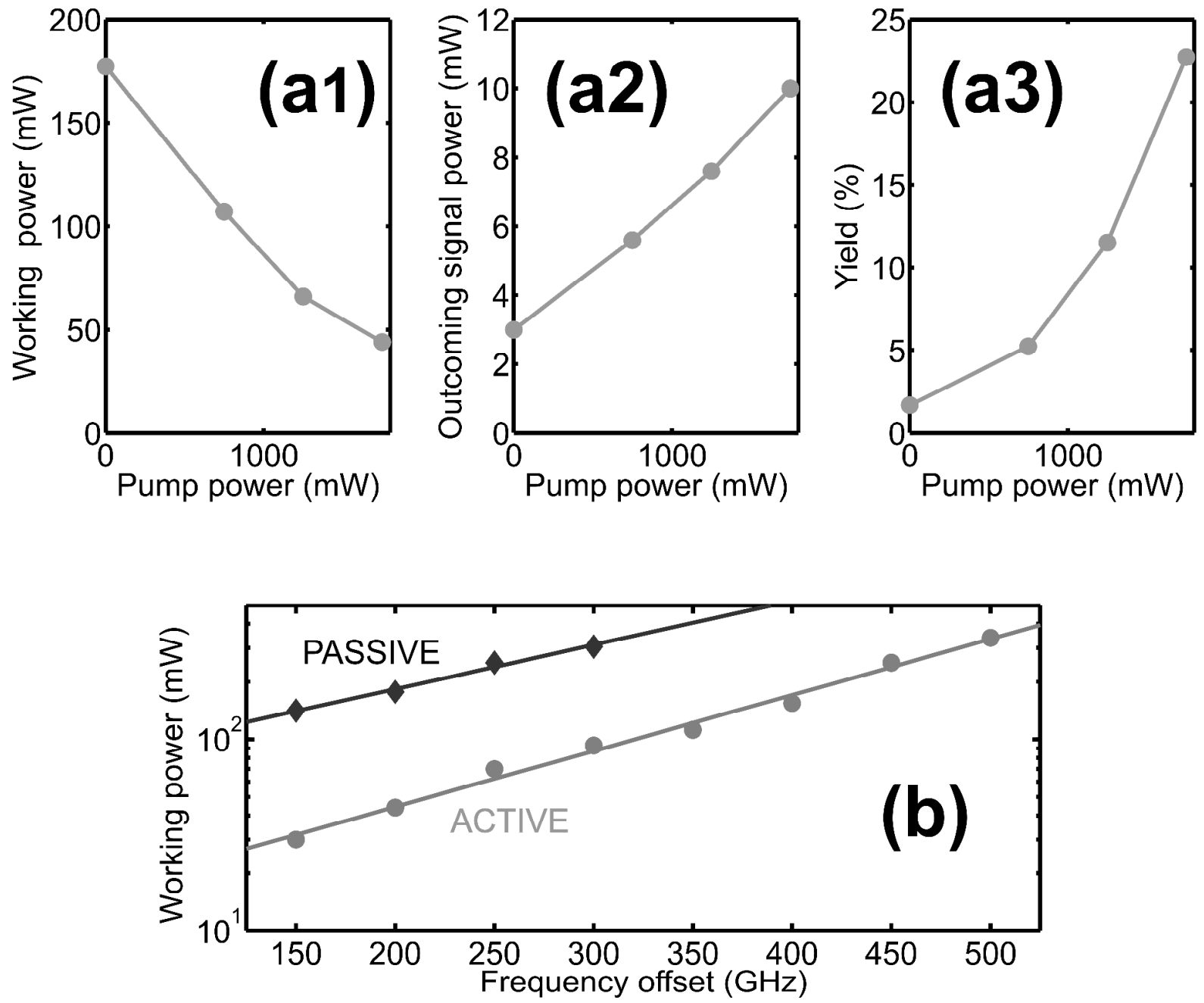
Figure 8

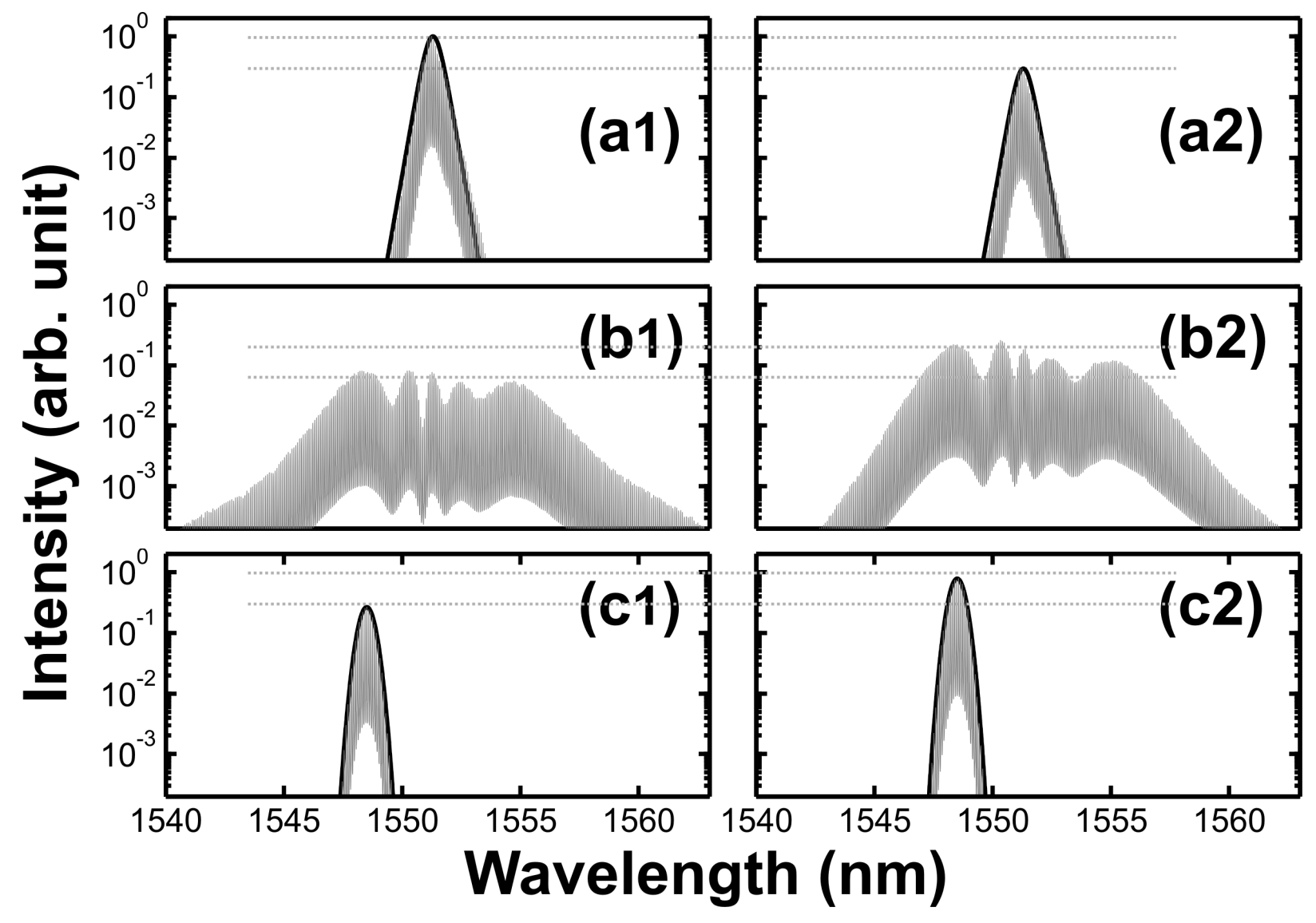


Figure 9
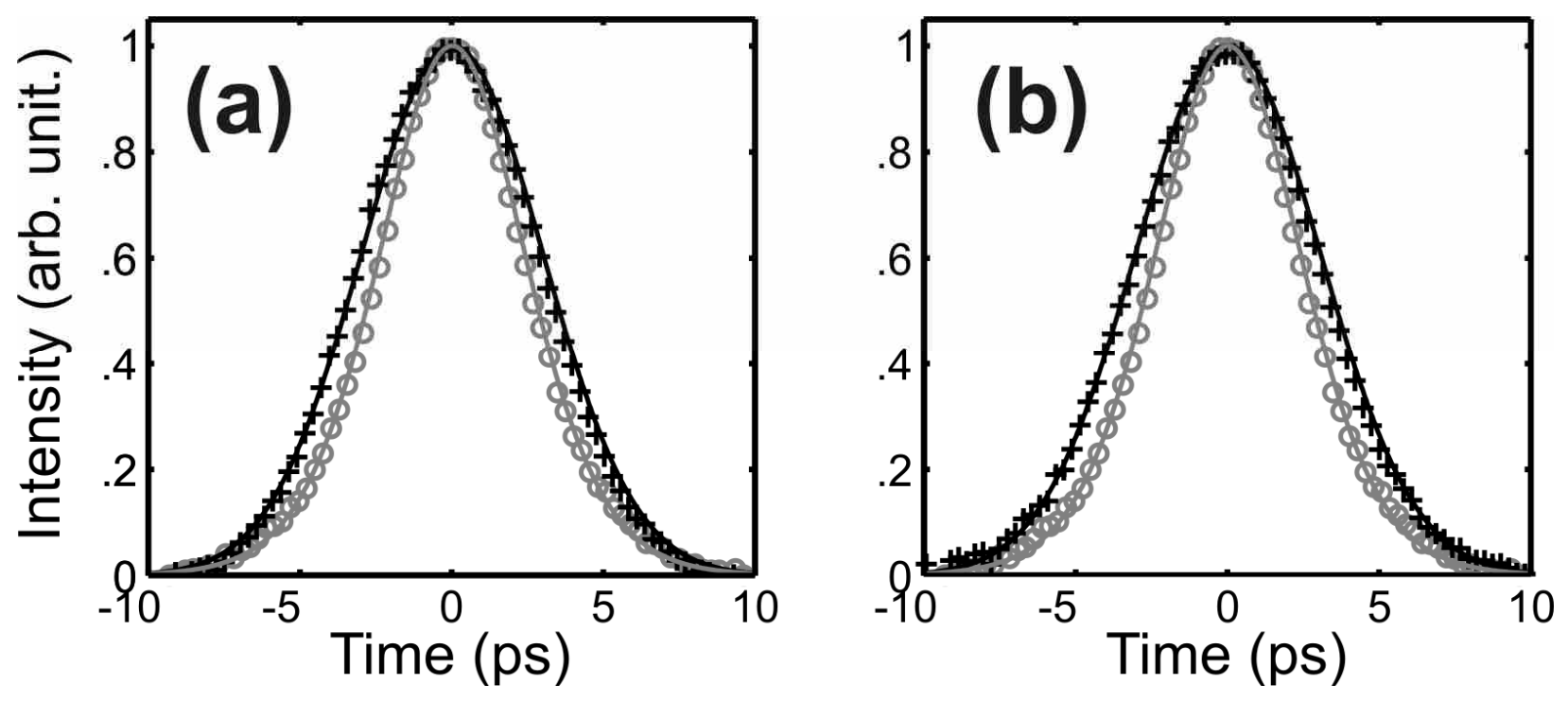
Figure 10
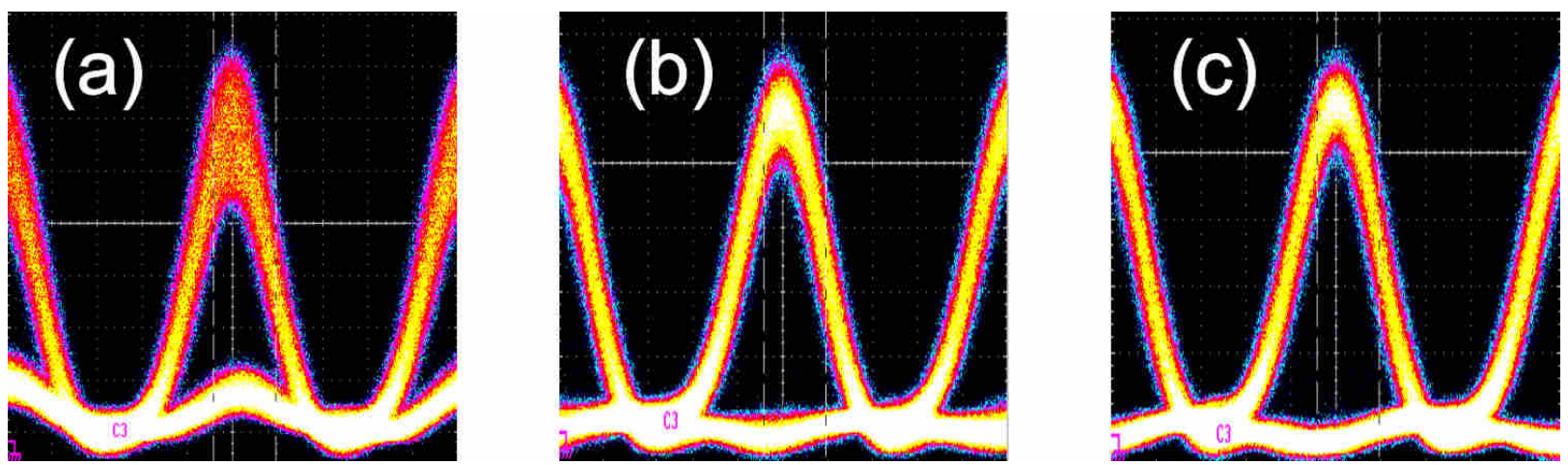